\title{
Retos Educativos y Psicosociales de las Prácticas Profesionales en el Área de Trabajo Social
}

\section{Educational and Psychosocial Challenges of Professional Practices in the Social Work Area}

\author{
Sandra Mireya Alturo Mendigaña ${ }^{1}$ \\ Universidad de La Guajira
}

\begin{abstract}
Resumen
El presente artículo establece una reflexión frente a los retos educativos y psicosociales que tienen los estudiantes y docentes orientadores de las prácticas profesionales en el área del trabajo social. Para tal efecto se llevó a cabo una investigación documental evaluativa derivada del análisis sociocritico, a partir una muestra de actividades, reportes e informes de práctica en las Instituciones de Educación Básica y Media donde han participado los estudiantes adscritos al Programa de Trabajo Social de la Universidad de La Guajira durante los años (2015-2016). Dentro de los principales hallazgos, se ha identificado que los escenarios de práctica profesional representan un amplio campo de diferentes problemáticas de orden educativo y de intervención psicosocial aplicada en los contextos relacionados a nivel escolar, familiar y comunitario. Dentro de las principales problemáticas se ha identificado los problemas de conflicto y violencia escolar, comunicación y desarrollo de habilidades socioeducativas, así como la incidencia de problemas de integración familiar, violencia y maltrato intrafamiliar, unidos a las necesidades de integración y desarrollo social comunitario.
\end{abstract}

Palabras clave: Practica profesional, educación, intervención psicosocial, trabajo social 


\begin{abstract}
This article establishes a reflection on the educational and psychosocial challenges facing the students and teachers who guide the professional practices in the area of social work. For this purpose, an evaluative documentary research derived from the socio-critical analysis was carried out, based on a sample of activities, reports and practice reports in the Basic and Media Education Institutions where the students assigned to the Social Work Program of the Universidad de La Guajira during the years (2015-2016). Among the main findings, it has been identified that professional practice scenarios represent a broad field of different educational problems and psychosocial intervention applied in the contexts related to school, family and community level. Among the main problems have been identified the problems of school conflict and violence, communication and development of socio-educational skills, as well as the incidence of problems of family integration, violence and intrafamily abuse, together with the needs of integration and community social development.
\end{abstract}

Keywords: Professional practice, education, psychosocial intervention, social work

\title{
Introducción
}

Las prácticas profesionales de los estudiantes son de gran importancia e impacto educativo y psicosocial, dado que cubren gran parte de las instituciones educativas públicas de la básica y la media en el Departamento de la Guajira. En particular, este es el caso de algunas Instituciones Educativas beneficiadas, tales como: Institución Etnoeducativa Aujero Chonkay, Institución Livio Reginaldo, Institución Educativa el Carmen, Institución Educativa Isabel María Cuesta, Centro de Educación Popular I.P.C., Institución Educativa María Doralisa, Institución Educativa Divina Pastora, Institución Educativa José Antonio Galán, Institución Educativa Helión Pinedo, Institución Educativa Celia Catalina e Institución Educativa Liceo Padilla, entre otras.

En esta instancia de la formación profesional, se ha adelantado una incorporación estratégica de innovaciones pedagógicas y psicosociales en el desarrollo de las prácticas de los estudiantes del programa de trabajo social, tomando como punto de partida un diagnostico situacional, enfocado al abordaje educativo y psicosocial de las diferentes problemáticas encontradas en cada una de las instituciones teniendo en cuenta que el trabajo se realiza en las jornadas que tenga la institución de lo cual los diagnósticos son diferentes de acuerdo a las situaciones y problemáticas encontradas.

El escenario de las prácticas profesionales, fortalece el desarrollo de competencias en interacción real y directa, lo cual implica una serie de retos curriculares para los docentes del área de Trabajo Social, orientados a la búsqueda permanente de estrategias, métodos y modelos de intervención que más se ajusten a la resolución satisfactoria de lo encontrado. Para tal efecto, se formuló un proyecto de intervención en trabajo social, con indicadores de seguimiento y monitoreo para el logro de las metas trazadas en los programas y actividades de la práctica profesional. 
Palomar (1993) plantea que el área de práctica profesional en trabajo social implica la necesidad de asumir un modelo sistémico de orden multidimensional en el cual el objeto de estudio implica comprender y articular las diferentes esferas y redes sociales que tienen las personas, con la finalidad de abordar integralmente los problemas. En tal sentido, se identifica que las prácticas profesionales tienen campos aplicados tales como las instituciones educativas, donde confluyen las dimensiones de la escuela, la familia y la comunidad.

Por su parte Guerra (2003), asume la importancia de la práctica desde el concepto de la instrumentalidad profesional orientada al servicio social, argumentando que:

La instrumentalidad del Servicio Social como mediación es el espacio para pensar en los valores subyacentes a las acciones, en el nivel y en la dirección de las respuestas que estamos dando y por las cuales, la profesión es reconocida o cuestionada socialmente. Es por su instrumentalidad que pasan las decisiones y alternativas concretas, de individuos concretos, en situaciones concretas. Las finalidades profesionales están inscriptas en un cuadro valorativo y solamente pueden ser pensadas en el interior de este cuadro, entendido como el acervo cultural del cual el profesional dispone y que orienta las elecciones teórico-metodológicas y ético-políticas, que a su vez, implican proyectar no solamente los medios/instrumentos de realización, sino también las consecuencias (p. 18).

En relación con las tendencias contemporáneas en Trabajo Social, Martíno y Bentura (2006) presentan sus aportes sobre el contexto uruguayo y se preguntan hasta qué punto el campo profesional se ha "desgastado". En tal sentido Martino, Bentura y Melgar (2006) se plantean los siguientes cuestionamientos:

¿Cuál será el futuro de nuestra profesión si los segmentos académicos no logran transformar las disposiciones intelectuales de las nuevas generaciones ante el conocimiento, en términos generales? ¿O no logran elaborar estrategias político-institucionales y pedagógicas que, a partir del perfil del estudiantado, permitan augurar el egreso de agentes capacitados que logren revertir los rasgos asociados a su extracción de clase? (p. 245).

Una de las principales problemáticas del contexto en el cual se desarrollan las prácticas en las Instituciones Educativas corresponde a la vulnerabilidad económica y las brechas relacionadas con el cubrimiento de las necesidades básicas en contextos de pobreza. Desde esta perspectiva Amar, Abello, Martínez, Monroy, Cortés y Crespo (2011) analizan las creencias sobre la naturaleza de la pobreza en niños y adolescentes. Dentro de sus principales conclusiones, los autores plantean que:

En las respuestas dadas por los niños participantes se evidenciaron las creencias |facilitadas por los agentes de socialización, asociadas a la predestinación de la pobreza. Esta idea se encontró particularmente en niños de NSE bajo, lo cual es propio del pensamiento fatalista que Martín Baró (1998) identificara en las comunidades vulneradas de Latinoamérica. El fatalismo se ha conocido tradicionalmente como la «aceptación resignada y pasiva de un destino irremediable emanado de alguna fuerza natural o de alguna voluntad sobrenatural» (Blanco \& Díaz, 2007, p. 3) que lleva a las personas a desvalorizar el poder que poseen para llevar las riendas de sus vidas, dificultando cualquier intento para alterar su situación (De la Corte, 1998), (p. 155). 
En este orden de ideas Castillo y Rodríguez (2016) en su estudio sobre la oferta educativa en el campo aplicado del trabajo social en Chile, plantean que:

En síntesis, la oferta educativa en términos de programas y matrícula impacta decididamente sobre los niveles de stock profesionales, hetereogeniza la labor formativa con disímiles requisitos de admisión y niveles formativos, y atenúan fuertemente las ya difusas fronteras laborales, en especial, aquellos límites que otrora distinguían y separaban lo técnico de lo profesional. Problemáticas cuya resolución se constituyen hoy por hoy en un desafío histórico sin precedentes para la disciplina del Trabajo Social (p. 50).

Dentro de los principales retos educativos que tienen las innovaciones pedagógicas y los procesos curriculares se encuentra el desarrollo de las competencias digitales para responder a las necesidades y expectativas contemporáneas de las prácticas profesionales en las diferentes áreas del conocimiento y en particular dentro del área del trabajo social.

En este tópico se encuentra como uno de los referentes centrales el estudio de Pinto, Cortés y Alfaro (2017) sobre el reto educativo de la transformación de la práctica docente a partir del Modelo Espiral de Competencias TICTACTEP. Este modelo genera una serie de interacciones funcionales entre las competencias de uso de las tecnologías de información y comunicación, con las competencias para el aprendizaje y el conocimiento, y en especial se considera el desarrollo de las competencias para el empoderamiento y la participación, las cuales son de vital importancia para fortalecer el desarrollo de las prácticas profesionales.

Este modelo tiene aplicaciones enfocadas al uso de herramientas tecnológicas tales como el E-Portafolio, el cual puede convertirse en un escenario innovador donde el docente y los estudiantes desarrollan de forma interactiva sus programas y actividades de práctica profesional. Al respecto Peña, Pinto y Atrio (2015), dentro de sus resultados sobre la implementación del E-Portafolio en las prácticas educativas, argumentan que:

Los resultados de orden cualitativo de esta investigación evidencian que los Eportafolios permiten valorar el progreso y mejoramiento de los participantes en torno a los estándares esperados en su ejecución desde el marco del desarrollo de competencias TICTACTEP. Se reconoce la pertinencia e impacto de la formación construccionista orientada al desarrollo de las competencias digitales con mayor apropiación de TIC y TAC, y la importancia estratégica que tiene el desarrollo de procesos continuos de formación docente (p.42).

En tal sentido Pinto, Cortés y Alfaro (2017) plantean que el Modelo Espiral de Competencias es una oportunidad que no solamente se orienta el desarrollo de las competencias de los estudiantes, sino que invita al docente a desarrollar sus competencias pedagógicas de forma digital, integrando las dimensiones TICTACTEP como medio para fortalecer el desarrollo social y comunitario en las prácticas profesionales. 


\section{Método}

El presente estudio tiene un abordaje de investigación documental evaluativa, orientado al análisis de los factores educativos, psicoeducativos y psicosociales derivados de las experiencias significativas que se han adelantado en el marco de las prácticas de formación profesional de los estudiantes del programa de Trabajo Social de la Universidad de La Guajira.

\section{Población y Muestra}

El marco poblacional y muestral objeto del presente estudio está conformado por las instituciones educativas en las cuales, los estudiantes del Programa de Trabajo Social han adelantado las prácticas profesionales, dentro de las cuales se pueden resaltar las siguientes: Institución Etnoeducativa Aujero Chonkay, Institución Livio Reginaldo, Institución Educativa el Carmen, Institución Educativa Isabel María Cuesta, Centro de Educación Popular I.P.C., Institución Educativa María Doralisa, Institución Educativa Divina Pastora, Institución Educativa José Antonio Galán, Institución Educativa Helión Pinedo, Institución Educativa Celia Catalina e Institución Educativa Liceo Padilla.

\section{Técnicas e Instrumentos}

Dada la naturaleza del estudio de corte evaluativo, se ha identificado como técnica la revisión sistemática y documental derivada de los reportes e informes de práctica de los estudiantes del Programa de Trabajo Social durante los últimos dos años (2015 - 2016).

\section{Procedimiento}

En primera instancia se seleccionaron los reportes e informes de práctica profesional y posteriormente se analizaron los mismos desde la perspectiva de las problemáticas y factores educativos y psicosociales asociados. Posteriormente se formularon matrices analíticas relacionadas con los aprendizajes y retos identificados en el análisis documental.

\section{Resultados}

En primera instancia, se han identificado elementos significativos que permiten establecer una nueva tendencia en el desarrollo de los procesos de apropiación social del conocimiento de los estudiantes, como parte de sus procesos de aprendizaje significativo, los cuales se enriquecen con la práctica en escenarios reales. A continuación se presenta en la siguiente tabla los principales problemas y retos identificados en el análisis documental. 
Tabla 1.

Sintesis de Ejes Problemáticas y Retos de las Prácticas Profesionales en Trabajo Social

\begin{tabular}{|c|c|c|}
\hline Eje & Principales Problemáticas & Retos de las Prácticas \\
\hline Escolar & $\begin{array}{l}\text { Conflicto Escolar, Violencia Escolar, } \\
\text { Bullying y Manifestaciones de } \\
\text { Acoso Escolar } \\
\text { Prácticas inadecuadas de castigo y } \\
\text { uso de la autoridad. } \\
\text { Deficit en el acceso a programas de } \\
\text { orientación vocacional y profesional } \\
\text { Desmotivación escolar, bajo } \\
\text { rendimiento académico y deserción } \\
\text { escolar. } \\
\text { Factores de riesgo asociados con } \\
\text { la salud sexual y reproductiva } \\
\text { (embarazo adolescente e infecciones } \\
\text { de transmisión sexual). }\end{array}$ & $\begin{array}{l}\text { Desarrollo de Programas de Intervención } \\
\text { Educativa y Psicosocial para el abordaje } \\
\text { integral de los problemas asociados con la } \\
\text { convivencia escolar bajo el marco de la Ley } \\
1620 . \\
\text { Desarrollo de Programas Psicoeducativos } \\
\text { orientados al fortalecimiento de las } \\
\text { competencias digitales para el mejoramiento del } \\
\text { rendimiento académico y la motivación escolar. } \\
\text { Desarrollo de procesos de integración de } \\
\text { las prácticas orientadas a la formulación de } \\
\text { proyectos de vida y la disminución del riesgo de } \\
\text { deserción escolar. } \\
\text { Desarrollo de programas orientados a promover } \\
\text { estilos de vida saludables con los niños(as) y } \\
\text { adolescentes }\end{array}$ \\
\hline Familiar & $\begin{array}{l}\text { Desintegración del núcleo familiar } \\
\text { Vulnerabilidad social y económica } \\
\text { de las familias } \\
\text { Violencia y maltrato intrafamiliar }\end{array}$ & $\begin{array}{l}\text { Diseño de programas de integración familiar } \\
\text { con las comunidades educativas. } \\
\text { Desarrollo de programas orientados a la } \\
\text { prevención integral de la violencia y el maltrato } \\
\text { intrafamiliar }\end{array}$ \\
\hline Comunitario & $\begin{array}{l}\text { Desarticulación entre los contextos } \\
\text { educativo, familiar y social de las } \\
\text { comunidades. } \\
\text { Vulnerabilidad social, económica y } \\
\text { ambiental de las comunidades. }\end{array}$ & $\begin{array}{l}\text { Desarrollo de programas y actividades que } \\
\text { promuevan la integración de las familias y } \\
\text { las instituciones educativas con sus entornos } \\
\text { comunitarios. } \\
\text { Formulación de proyectos comunitarios } \\
\text { orientados al desarrollo sostenible. }\end{array}$ \\
\hline
\end{tabular}

Fuente. Elaboración propia.

En relación con el análisis de las experiencias y la percepción que tienen las Instituciones Educativas, cabe resaltar que los coordinadores, han expresado su satisfacción y agradecimiento por el trabajo realizado, estos a su vez emiten evaluaciones formales donde les asignan notas altas a los estudiantes por su eficaz desempeño reconocimientos al programa de Trabajo Social por el aporte social que está desarrollando en el departamento, con el fin de aportar a la convivencia sana y en paz de las comunidades.

\section{Discusión}

Los resultados son consistentes con las reflexiones de Castillo y Rodríguez (2016) sobre los factores asociados con la oferta educativa en el área de trabajo social, resaltando las dificultades, necesidades y expectativas de vinculación profesional en el contexto chileno. Dentro de sus aportes, se resalta que el Trabajo Social por su naturaleza es uno de los campos de mayor relevancia y pertinencia para el desarrollo humano, social y sostenible de las comunidades.

El Modelo TICTACTEP presenta una serie de retos educativos que a su vez se orientan al mejoramiento de las competencias digitales y los procesos de aprendizaje significativo apoyados en herramientas como el E-Portafolio (Peña, Pinto y Atrio, 2015). 
Adicionalmente se identifica el reto educativo referido al desarrollo de estrategias psicopedagógicas ajustadas a las necesidades de los niños y adolescentes tal como lo analizan Antequera-Manotas y Cortés-Peña (2016) y al abordaje de las problemáticas psicosociales que pueden incidir en la interacción escolar y el rendimiento académico de los estudiantes, generando nuevas oportunidades de aprendizaje significativo, tal como reportan Avendaño Villa, Cortés Peña y Guerrero Cuentas (2015).

En respuesta a las tendencias del área del Trabajo Social y sus oportunidades de redireccionamiento estratégico a nivel curricular y profesional, el presente estudio resalta la necesidad de desarrollar estudios de orden nacional e internacional orientados a formular las perspectivas de competencias profesionales y competencias específicas necesarias para que los futuros profesionales respondan y aporten al desarrollo social e integral de sus comunidades. En este sentido se resaltan los planteamientos de Martino, Bentura y Melgar (2006):

Profundizar el estudio de nuestra profesión y la necesaria construcción de un proyecto profesional colectivo y consensuado, abierto a los debates y experiencias regionales, son condiciones sine qua non para poder desarrollar la investigación, la producción de conocimientos o saberes, y una práctica profesional autónoma, técnicamente pertinente y éticamente sustentada. Sin ello, aún en una nueva institucionalidad académica, Trabajo Social no podrá desarrollar investigación sustantiva, producir conocimientos ni desplegar estrategias de acción que no vayan más allá de la simple falsificación de la verdad de sus objetos y procedimientos (p. 246).

A partir de la investigación evaluativa de corte documental, también se identificó que el proceso de la práctica profesional requiere cada vez más de la integración de saberes y por lo tanto se requiere fortalecer el desarrollo de competencias para el trabajo en equipo y el desarrollo de procesos de prácticas integradoras de orden interdisciplinario, donde las Instituciones Educativas se beneficien el aporte que las diferentes áreas tales como pedagogía, psicología, trabajo social puedan generar a su vez equipos de practicantes interdisciplinarios, para responder a los retos educativos y psicosociales.

Por lo tanto, los retos de las prácticas y el desarrollo de las competencias profesionales en el área del trabajo social, implican ir más allá de la mirada asistencial hacia el abordaje integral de las problemáticas sociales, ambientales y económicas de las comunidades, más allá de las tendencias de masificación y atomización de programas en la Educación Superior, tal como lo plantea Rama (2009).

\section{Referencias}

Amar Amar, J., \& Abello Llanos, R., \& Martínez González, M., \& Monroy Agamez, E., \& Cortés Peña, O., \& Crespo Romero, F. (2015). Beliefs about poverty related to social categorization in childhood. Suma Psicológica, 22 (1), 9-17.

Amar, J., \& Abello, R., \& Martínez, M., \& Monroy, E., \& Cortés, O., \& Crespo, F. (2011). Creencias sobre la naturaleza de la pobreza en un grupo de niños de nivel socioeconómico alto y bajo de la ciudad de Barranquilla (Colombia). Psicología desde el Caribe, 27, 136-159.

Amar, J., \& Abello, R., \& Martínez, M., \& Monroy, E., \& Cortés, O., \& Crespo, F. (2011). Categorización social y cogniciones infantiles sobre la pobreza en niños: Una mirada desde el esencialismo psicológico. Investigación \& Desarrollo, 19 (1), 116-139. 
Antequera-Manotas, E., \& Cortés-Peña, O. (2016). Problems, challenges, trends and prospects for special education for talented children. The European Proceedings of Social \& Behavioural Sciences-EpSBS, 16(1), 857-861.

Avendaño-Villa, I., Cortés-Peña, O. F., \& Guerrero-Cuentas, H. (2015). Competencias sociales y tecnologías de la información y la comunicación como factores asociados al desempeño en estudiantes de básica primaria con experiencia de desplazamiento forzado. Diversitas, 11(1), 13-36.

Castillo Riquelme, V., \& Rodríguez Garcés, C. (2016). Los problemas del stock en campos profesionales difusos: Oferta educativa en Trabajo Social. Estudios Pedagógicos, XLII (1), 37-52.

Curbelo-Hernández, E., \& Del-Sol-Florez, H. (2010). Trabajo social y mediación familiar: un enfoque para la protección del menor en el proceso mediador. Orientaciones para la práctica profesional en los supuestos de ruptura de pareja. Portularia, $X$ (2), 33-49.

Falla Ramírez, U., \& Gómez Contreras, S., \& Rodríguez, R. (2013). Fenomenología de motivos en la resolución de dilemas que enfrentan los trabajadores sociales en su práctica profesional. Tabula Rasa, 18, 289-299.

Fallas Jiménez, Y. (2009). Trabajo social, formación profesional y categoría trabajo. Reflexiones, 88 (1), 67-76.

Gómez-Gómez, F. (2010). Competencias profesionales en trabajo social. Portularia, X (2), 51-63.

Guerra, Y. (2003). Instrumentalidad del proceso de trabajo y Servicio Social. Revista Servicio Social \& Sociedad, 62, 5-15.

Iturrieta Olivares, S. (2012). Desafíos del Trabajo Social en un campo laboral con límites profesionales difusos. Revista Katálysis, 15(2), 163-172.

Mallardi, M., \& Cañizares, B. (2011). La aprehensión de la cuestión social en la práctica del Trabajo Social: Aportes para la elaboración de proyectos sociales en el ejercicio profesional. Portularia, XI (2), 15-21.

Martino Bermúdez, M., \& Bentura, C., \& Melgar, A. (2006). Tendencias actuales en el Trabajo Social uruguayo: hacia un campo profesional envejecido?. Revista Katálysis, 9 (2), 237-248.

Palomar Villena, M., \& Suárez Soto, E. (1993). El modelo sistémico en el trabajo social familiar: consideraciones teóricas y orientaciones prácticas. Alternativas. Cuadernos de Trabajo Social, 2,169-184.

Peña, O. F. C., Santos, A. R. P., \& Atrio, S. I. (2015). E-portafolio como herramienta construccionista del aprendizaje activo en tecnología educativa. Revista Lasallista de Investigación, 12(2), .

Pinto Santos, A., \& Cortés Peña, O., \& Alfaro Camargo, C. (2017). Hacia la transformación de la práctica docente: modelo espiral de competencias TICTACTEP. Pixel-Bit. Revista de Medios y Educación, 51, 37-51.

Ponce-de-León-Romero, L. (2012). Teorizar la experiencia profesional del trabajo social. Portularia, XII, 141-147.

Rama, C. (2009). La tendencia a la masificación de la cobertura de la educación superior en América Latina. Revista Iberoamericana de Educación, 50, 173-195.

Ronda Ortín, L. (2012). El educador social. Ética y práctica profesional. Pedagogía Social. Revista Interuniversitaria, 19, 51-63. 